\title{
Design and implementation of Adaptive Content Trisection System based on SCORM
}

\author{
Yang Rui ${ }^{1, a}$, Fan WenJie ${ }^{1, b}$ \\ ${ }^{1}$ China Meteorological Administration Training Centre, 10081 Beijing,China \\ ayangr@cma.gov.cn, ${ }^{\text {b }}$ fanwj@cma.gov.cn
}

Keywords:SCORM;Trisectionsystem; Adaptive content

\begin{abstract}
This paper proposes a new adaptive content trisection systembased on SCORM to solve the problems in traditional trisection system such as lengthybrowsingtime, lack of content point structure, interaction andbrowsing process tracking. The newly designed trisection systemset up a new content aggregation through designs of adaptive content trace and controllablebrowsing process. Three models based on SCORM, CAM, RTE and SN, are adopted in this paper to implement the new adaptive content trisection system.
\end{abstract}

\section{Introduction}

Trisection Systemis one of the most famous multimedia online systems which consists of video, PPT, and index section. While it often has a longer browsingtime and a single linear information transmission which may let the systembe lack of interactionand the userbe tired of browsing so that the efficiency of trainingisgreatly reduced. SCORM (Sharable Content Object Reference Model) as one of the most popular technical specifications model provides a set of common and systematic organization of the curriculum and data transmission specification that realizes a highlycontrollablein the browsing process. This paper implements a new adaptive content trisection systemby optimizing and upgrading the traditionaltrisection systembased on SCORM.

\section{SCORM}

CAM:content aggregation model,is used to describe the structure of the browsingresources by the manifest file inan XML file.

RTE: run time environment, is presented for the browsing materials to send and receiveinformation between the user and the LMS by API and Data Model.

SN: Sequencingand Navigation, allows thecreator to create a contentat design time that behaves in a user-centricway.

\section{Design of Adaptive Content Trisection System}

Traditional trisection systemis subject to missing content points and administrationout of control because of distracted content points, content without key points and lengthybrowsing time which usually takes more than 45 minutes.This paper proposes optimized adaptive content trisection system by designs of content aggregation, adaptive content trace and controllablebrowsing process. This leads to monitor browsing process in realtime and guideeffectivebrowsing.

Design of Content Aggregation.We have to reorganize thecontent points of original system and divide them into reasonable classes according torequirement. Then we aggregate them to a new activity tree. This paper split a standard system of 45 minutes into five parts of 9 minutes as an independent unit which is divided into three partsmoduleincluding basic module, general module and advanced module.The Adaptive Content Trisection System Activity Tree as shown in Figure 1: 


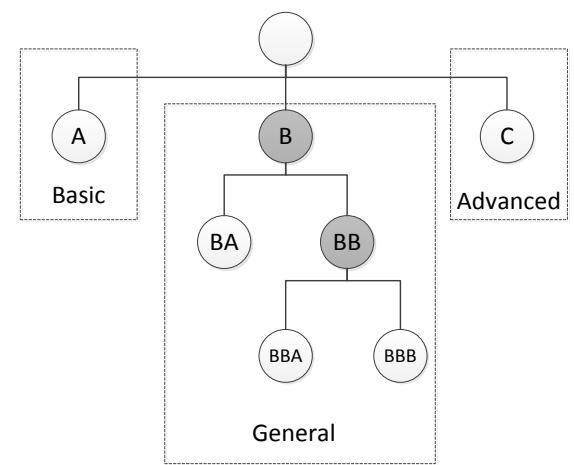

Figure 1Adaptive Content Trisection System Activity Tree

Design of Adaptive Content Trace.According to the activity tree, we designpre-test and post-test unit to intelligentguide the userin adaptive browsing paths, which breaks the traditional single linear browsingmode. We assumea linear model among A, B and C module andchoice model insideB module in which the user must attempt the pre-testfirst. If the userpasses the pre-test, he can skip BA. In BB moduletheuser can select the child activities freely. After the userfinishes the unit, he must take a post-test. If the userfails in the post-test, hemust retry BA. The adaptivecontent trace as shown in Figure 2:

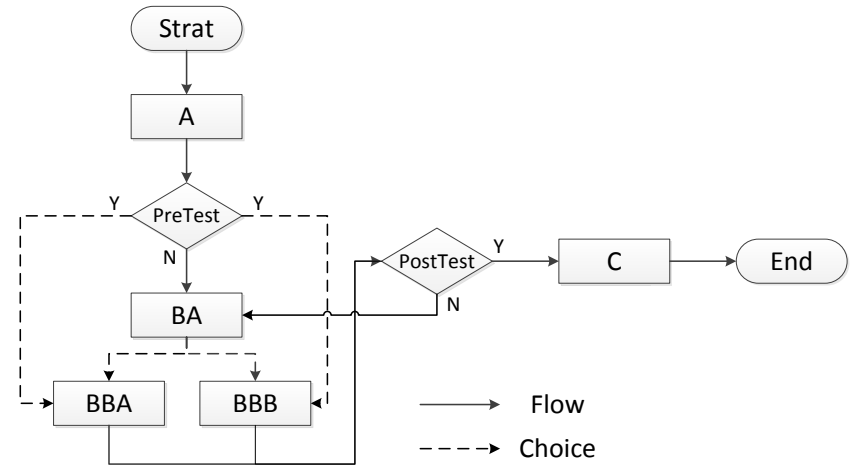

Figure 2Adaptive Content Trace

Design of Controllable BrowsingProcess. We control the behavior of users and record browsingtime, exit time points and other key informationby RTE whichprovide user with the opportunity to know about his browsing progress and to guide him continueat last time point. This also gives the administers a chance tomaster, evaluate and analyze the whole picture of all users. RTEs primary data is shown in the following table:

\begin{tabular}{|l|l|}
\hline \multicolumn{1}{|c|}{ Data Model } & \multicolumn{1}{c|}{ Behavior Description } \\
\hline cmi.max_time_allowed & Maximun Time Allowed to control browsing time \\
\hline cmi.location & Location to record the exit time point \\
\hline cmi.session_time & Session Time to record the browsing time \\
\hline cmi.objectives & $\begin{array}{l}\text { Objectives to content working with other elements } \\
\text { in SN }\end{array}$ \\
\hline
\end{tabular}

\section{Implementation of Adaptive Content Trisection System}

(1)We aggregate content to a new adaptive content trisection system by the elements called organizationsthat defines a new content tree and resourcesthat defines the resource in CAM. The contentunits and test unitsare defined as the SCO objects to facilitate subsequent controlled browsing process. Key code is shown in the following:

$<$ organizations $>$
$\quad<$ organization identifier="Adaptive Content
Trisection System">
$<$ item identifier="A"identifierref="rA"/>
$<$ item $\quad$ identifier="PreTest"identifierref="rPr

$<$ resources $>$

$<$ resource

identifier="rA"adlcp:scormtype="sco"href="rA.ht $\mathrm{m} " />$

$<$ resource

identifier="rBA"adlcp:scormtype="sco"href="rBA. 


\begin{tabular}{|c|c|}
\hline $\begin{array}{l}\text { eTest"/> } \\
<\text { item identifier="B"> } \\
<\text { item } \\
\text { identifier="BA"identifierref="rBA"/> } \\
<\text { item identifier="BB"> } \\
\quad<\text { item } \\
\text { identifier="BBA"identifierref="rB } \\
\text { BA"/> } \\
\quad<\text { item } \\
\text { identifier="BBB"identifierref="rB } \\
\text { BB"/> } \\
</ \text { item }> \\
</ \text { item }> \\
<\text { item } \\
\text { identifier="PostTest"identifierref="rPo } \\
\text { stTest"/> } \\
<\text { item } \\
\text { identifier="C"identifierref="rC"/> } \\
<\text { /organization> } \\
\text { /organizations }>\end{array}$ & $\begin{array}{l}\text { htm"/> } \\
\text { <resource } \\
\text { identifier="rBBA"adlcp:scormtype="sco"href="rB } \\
\text { BA.htm"/> } \\
\text { <resource } \\
\text { identifier="rBBB"adlcp:scormtype="sco"href="rB } \\
\text { BB.htm"/> } \\
\text { <resource } \\
\text { identifier="rC"adlcp:scormtype="sco"href="rC.htm } \\
\text { "/> } \\
\text { <resource identifier="rPreTest } \\
\text { "adlcp:scormtype="sco"href=" rPreTest.htm"/> } \\
\text { <resource identifier="rPostTest } \\
\text { "adlcp:scormtype="sco"href=" rPostTest.htm"/> } \\
\text { </resources> }\end{array}$ \\
\hline
\end{tabular}

(2)We define the rules among modules by respectivelyinserting a SN element called sequencing in the Organization and Bmodule which define the controlModeis forwardOnly= "true" and choice= "true", that is, browsing order is a linear modeamong modules and free selection mode inside the B module.

(3)We defineaobjective obBA and map it to the globalobjective obPreTest by inserting a element called sequencing in the BA module.obBA's success condition is defined as test scores is greater than $60 \%$.If the user cannot satisfy the objective, BA module will be skipped. Key code is shown in the following:

<imsss:sequencing>

$<$ imsss:sequencingRules $>$

<imsss:preConditionRule>

<imsss:ruleConditionsconditionCombination="all">

<imsss:ruleConditionreferencedObjective="obBA" condition="objectiveMeasureGreaterThan" measureThreshold="0.59"/>

</imsss:ruleConditions >

<imsss:ruleAction action="skip" / >

$<$ /imsss:preConditionRule>

$<$ imsss:sequencingRules $>$

<imsss:objectives>

<imsss:primaryObjectiveobjectiveID="obBA">

<imsss:mapInfotargetObjectiveID="obPreTest" readSatisfiedStatus="true"/>

</imsss:primaryObjective $>$

$<$ imsss:objectives $>$

</imsss:sequencing>

(4)Similarly, we definea objective obBB and map it to the globalobjective obPostTest by inserting a element called sequencing in the BB module.obBB's success condition is defined as test 
scores is greater than $80 \%$ and it's postConditionRule is action="retry". If the user cannot satisfy the objective, BA module must be retried. Key code is shown in the following:

\section{Conclusion}

This paper has explored a new adaptive content trisection systembased on SCORM by reasonable splitting and aggregation. It has improved the efficiency of browsing and made a controllableand personalized browsing into reality.

\section{References}

[1] ADL SCORM, Sharable Content Object Reference Model(SCORM) 2004 2nd Edition,http://www.adlnet.gov/downloads/70.cfm

[2] The RELOAD Project: (The RELOAD Metadata and Content Packaging Editor), http://www.reload.ac.uk/editor.html.

[3] Li Qing, Research on learning activity model, doctoral dissertationof East China Normal University, April 2005, pp.77-90

[4] IMS Simple Sequencing BestPractice and ImplementationGuide,http://www.imsglobal.org/simplesequencing/ssv1p0/imsss_bestv1p0.html.

[5] Learning System Architecture Lab. Content developers' guide toIMS Simple Sequencing. Carnegie Mellon University, SomeRights Reserved. 2003, pp.7-8

[6] Roderick A. Farmer and Baden Hughes “A Situated Learning Perspective on Learning Object Design,” ICAL2005 Proceeding, p7 